\title{
Compensation and Weights for Trade-offs in Engineering Design: Beyond the Weighted Sum
}

\author{
Michael J. Scott ${ }^{1}$ \\ Assistant Professor \\ e-mail: mjscott@uic.edu \\ Department of Mechanical \& Industrial \\ Engineering, \\ University of IIlinois at Chicago, Chicago, IL
}

Erik K. Antonsson ${ }^{2}$

Professor

Engineering Design Research Laboratory,

Division of Engineering and Applied Science,

California Institute of Technology, Pasadena, CA
Multicriteria decision support methods are common in engineering design. These methods typically rely on a summation of weighted attributes to accomplish trade-offs among competing objectives. It has long been known that a weighted sum, when used for multicriteria optimization, may fail to locate all points on a nonconvex Pareto frontier. More recent results from the optimization literature relate the curvature of an objective function to its ability to capture Pareto points, but do not consider the significance of the objective function parameters in choosing one Pareto point over another. A parametrized family of aggregations appropriate for engineering design is shown to model decisions capturing all possible trade-offs, and therefore can direct the solution to any Pareto optimum. This paper gives a mathematical and theoretical interpretation of the parameters of this family of aggregations as defining a degree of compensation among criteria as well as a measure of their relative importance. The inability to reach all Pareto optima is shown to be surmounted by this consideration of degree of compensation as an additional parameter of the decision. Additionally, the direct specification of importance weights is common to many decision methods. The choice of a single point from a Pareto frontier by specifying importance weights alone is shown to depend on the degree of compensation implicit in the aggregation. Thus both the degree of compensation and weights must be considered to capture all potentially acceptable decisions. A simple truss design example is used here to illustrate the concepts. [DOI: 10.1115/1.1909204]

Keywords: Multicriteria Analysis, Engineering Design, Design Decision Making, Aggregation Functions, Functional Equations, Compensation, Importance Weights, Indifference, Utility

\section{Introduction}

Multicriteria decision making is an important part of engineering design. There are many methods, both informal and formal, that support such design decision making, including quality function deployment (QFD) [1], and the analytic hierarchy process (AHP) [2]. These design decision methods share several key features. All rely on the aggregation of multiple criteria or measures of performance to choose among designs, and most methods allow for the assignment of importance to individual attributes through the use of weights. These importance weights are intended to allow for meaningful comparison of many options when two or more attributes must be traded off against each other. Among decision methods, weighted-sum aggregation of preferences is by far the most common, as is direct specification of importance weights.

Although the problems of the weighted-sum formulation for optimization have been discussed previously [3-8], that discussion has been centered on the optimization task of recovering an entire Pareto frontier by adjusting parameters of the objective function, not on the decision task of selecting parameters to achieve a desired outcome. This paper gives an interpretation of the decision parameters as governing both the relative importance of the attributes and the degree of compensation between them. Compensation refers to a willingness to allow high performance on one attribute to compensate for low performance on another and is a property of a decision rather than a design. Mirroring

\footnotetext{
${ }^{1}$ Corresponding author.

${ }^{2}$ Chief Technologist of NASA's Jet Propulsion Laboratory.

Contributed by Design Theory and Methodology Committee for publication in the Journal of Mechanical Design. Manuscript received December 19, 2003; revised received January 17, 2005. Associate Editor: Clive L. Dym.
}

known results from optimization, it is shown that any method that relies exclusively on importance weights to define a decision runs the risk of missing "optimal" options. A complete model of a two-attribute decision requires an additional parameter to specify the degree of compensation between criteria [9]. The ability to adjust the degree of compensation to capture all Pareto points is related to prior results concerning the ability to adjust the curvature of an objective function [6]. However, since the decision goal is not to capture all Pareto points, but to select desired points, it is also shown here that even when all Pareto points can be selected, the choice of one point from a Pareto set is influenced not only by the importance weights, but also by the degree of compensation. Thus the direct specification of importance weights can only be an ad hoc process, producing answers that are often inappropriate. Several relevant results are presented here:

- The degree of compensation between criteria is a crucial parameter that defines engineering design decisions. In some cases, the good performance of one attribute can compensate, to some degree, for the poor performance of other attributes. Both the degree of compensation and the relative importance weighting among criteria must be specified to define a decision.

- For decision making that conforms to the axioms of rational design $[10,11]$, a parametrized family of functions, with compensation parameter $s$, was previously shown to span a complete range of degrees of compensation [12].

- The compensation parameter $s$ increases with the degree of compensation. This is interpreted mathematically in two different ways, most importantly by showing that higher degrees of compensation favor designs with high performance on one attribute and low performance on another over designs with solid but unexceptional performance on both at- 


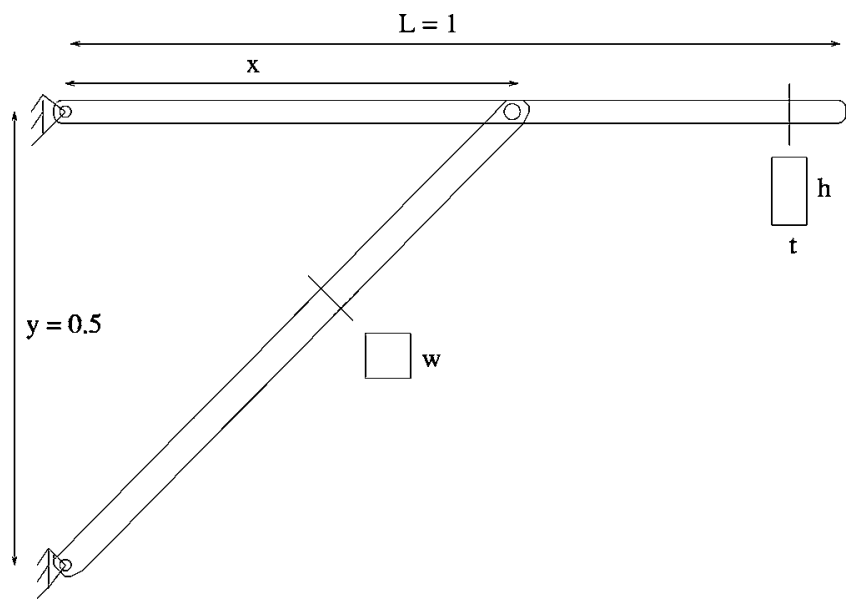

Fig. 1 Example of a simple truss structure

tributes. The compensation parameter must be taken together with the importance weights to define a decision. The degree of compensation between attributes, along with the relative importance weights, is a trade-off strategy.

- The use of a weighted sum preselects a single degree of compensation between attributes in which the parameter $s$ $=1$. It is thus incapable of modeling all possible trade-off strategies.

- When employing this parametrized family of functions, a ratio of weights and a degree of compensation to select any Pareto optimal point can always be found.

- The ability to choose any Pareto point is not present when the degree of compensation is preselected, as, for example, with the use of a weighted sum.

\section{Motivating Example: A Simple Truss Structure}

Consider the structure shown in Fig. 1. This is a pin-jointed two-member bracket to support a load of $1 \mathrm{~kg}(P=1 \mathrm{~kg})$ at a distance of $1 \mathrm{~m}$ from a wall $(L=1)$. The positions of the wall mounts are fixed, with the lower support $0.5 \mathrm{~m}$ below the upper support $(y=0.5)$. Both members are made of aluminum (6061T6). The designer controls four design variables

$x \in[0.1 \mathrm{~m}, 0.9 \mathrm{~m}]$ distance from wall to pin $t \in[5 \mathrm{~mm}, 20 \mathrm{~mm}]$ thickness of bending member

$h \in[5 \mathrm{~mm}, 20 \mathrm{~mm}]$ height of bending member

$w \in[5 \mathrm{~mm}, 20 \mathrm{~mm}]$ width of (square) compression member

For this example, the performance measures to consider are the mass $M$ of the structure and the safety factor $S$. Both quantities can be expressed analytically, but in this example the performance calculation will be treated as a black box. The details of the calculations are presented below. The design problem is to minimize the mass of the structure while maximizing the factor of safety.

Furthermore, suppose that no additional advantage is gained from factors of safety above 10 . Also, designs with safety factors below 1 should not be considered. Using optimization or other means, it can be determined that the minimum mass achievable with a factor of safety of 1 is $123 \mathrm{~g}$, while the minimum mass achievable with a factor of safety of 10 is $302 \mathrm{~g}$. The best designs will be trade-offs between the safety factor and the mass.

Assuming that both $123 \mathrm{~g}$ (the lowest possible mass for the acceptable range of safety factors) and $302 \mathrm{~g}$ (the mass corresponding to the highest safety factor) are acceptable, it is common to normalize the performance measures. Here we follow the approach of the method of imprecision (MoI) $[9,13]$ and normalize by specifying preferences. The results would not be substantially
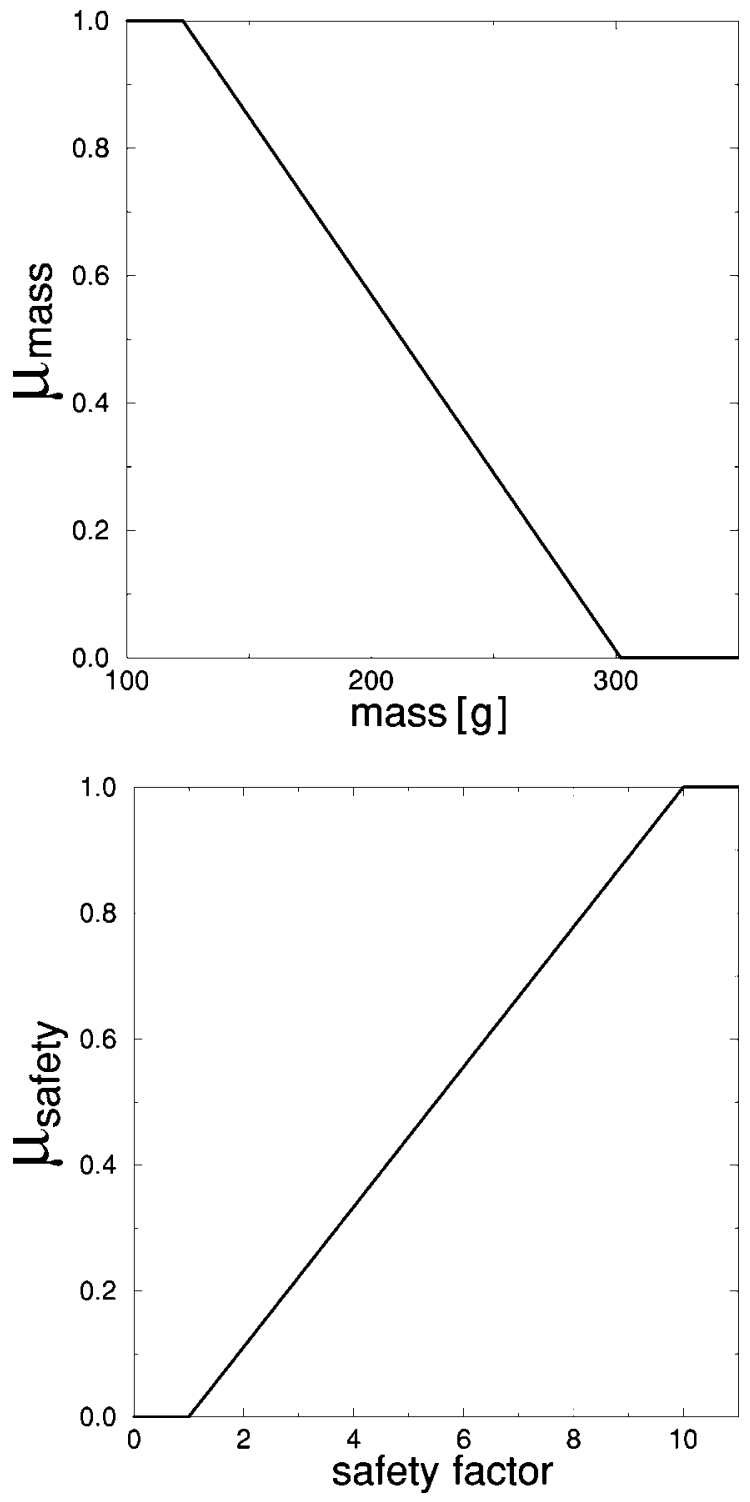

Fig. 2 Preferences for mass and safety factor

different for other normalization schemes; indeed, normalization is not necessary. Let the preferences for mass and safety be as follows:

$$
\begin{gathered}
\mu_{\text {mass }}(M)=\frac{302-M}{179} \\
\mu_{\text {safety }}(S)=\frac{S-1}{9}
\end{gathered}
$$

so that $\mu_{\text {mass }}(123)=1, \quad \mu_{\text {mass }}(302)=0, \quad \mu_{\text {safety }}(1)=0, \quad$ and $\mu_{\text {safety }}(10)=1$, as shown in Fig. 2 . Note that these simple linear preferences are chosen for convenience; all the results presented here hold for more complicated preferences as well.

One way to select a best design is to assign importance weights to the two criteria and then to use a weighted sum to aggregate preferences; the best designs will have the highest overall preference. Let the importance weights assigned to mass $\left(\omega_{1}\right)$ and safety $\left(\omega_{2}\right)$ be normalized so that their sum is 1. Employing this approach, there are only three possible "best" points, summarized in Table 1. According to this weighted-sum aggregation, all other possible points are worse when both mass and safety factor are considered. These points are shown on the graph in Fig. 3. 
Table 1 Three "best" possible points found with a weighted sum

\begin{tabular}{ccccccc}
\hline \hline$\omega_{1}$ & $\begin{array}{c}\text { Mass } \\
(\mathrm{g})\end{array}$ & $\begin{array}{c}\text { Safety } \\
\text { factor }\end{array}$ & $x$ & $t$ & $h$ & $w$ \\
\hline$\omega_{1}>0.658$ & 123 & 1 & 0.71 & 5 & 5 & 5 \\
$0.492<\omega_{1}<0.658$ & 129 & 1.6 & 0.82 & 5 & 5 & 5 \\
$\omega_{1}<0.492$ & 302 & 10 & 0.9 & 5 & 9.34 & 8.16 \\
\hline \hline
\end{tabular}

The three "best" points shown in Fig. 3 do not represent the entire range of reasonable trade-offs between the two performance measures, mass and safety. To make the notion of a "reasonable" decision more precise, we use the idea of Pareto optimality [14]:

Definition 1. The alternative $\mathrm{A}$ dominates the alternative $\mathrm{B}$ if $\mathrm{A}$ performs no worse than $\mathrm{B}$ on all attributes, and better than $\mathrm{B}$ on at least one attribute. In this case, regardless of the weights or the degree of compensation, it is always better to choose A over B. A feasible solution is undominated (or Pareto optimal) if there is no other feasible solution that dominates it.

Figure 4 shows a plot of all the feasible Pareto optimal points, normalized with respect to preference, with the three points from Fig. 3 retained. (The calculation of the Pareto frontier is detailed

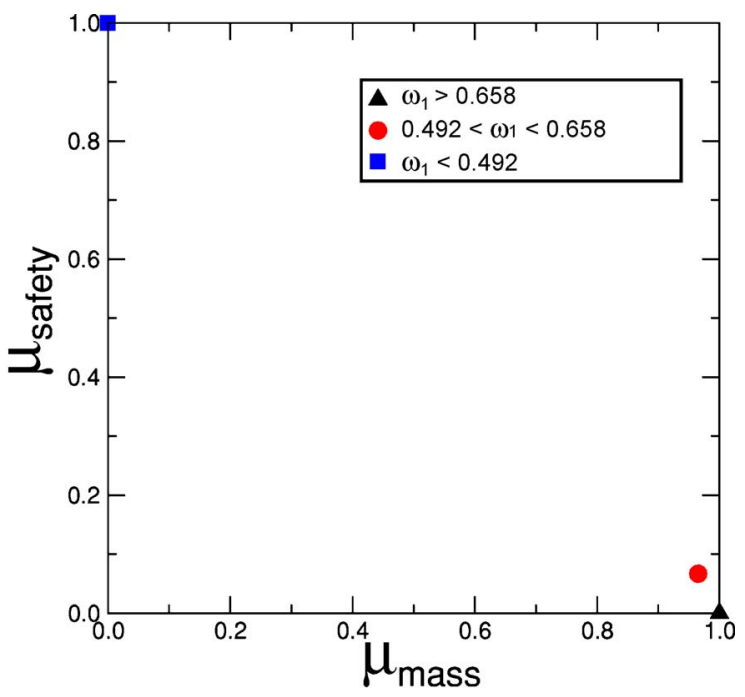

Fig. 3 Three "best" points found using weighted-sum exploration

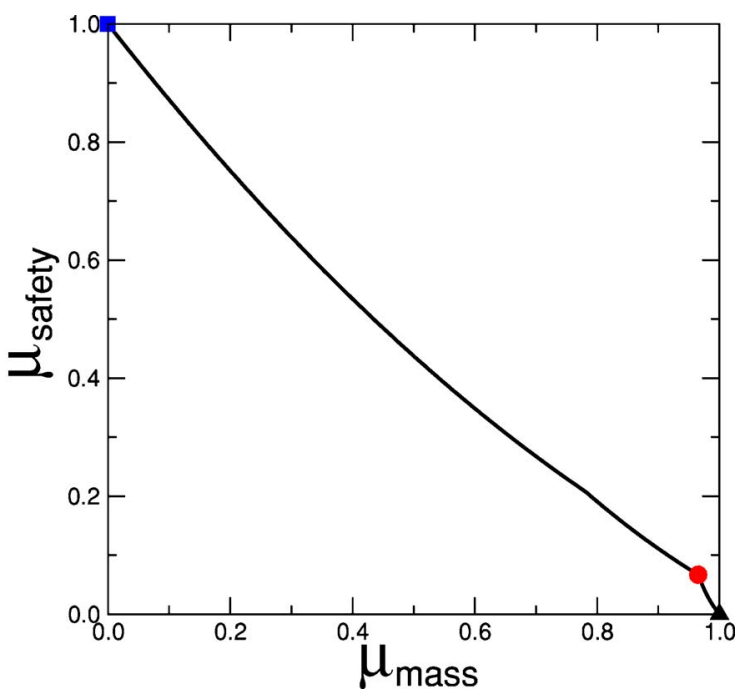

Fig. 4 Pareto frontier with three "best" points in Sec. 6.) Examining the entire Pareto frontier, we see that it is made up of two concave sections. The weighted-sum approach fails to identify any Pareto points on the concave sections of the frontier, though it is quite possible that one of those points represents the most desirable compromise.

This inability of the weighted sum to generate an entire (concave) Pareto frontier has long been known in an optimization context. Koski [3] called this deficiency "well known" and argued that such concave Pareto frontiers are common in structural optimization. Messac [4] presented the physical programing paradigm as an alternative to weighted-sum methods, and Chen et al. [15] advocated a Tchebycheff norm to capture all Pareto points. Athan and Papalambros [5] showed that a weighted compromise programing function could capture an entire Pareto frontier, given a suitable choice of exponents. Messac et al. [6,7] and Messac and Ismail-Yahaya [8] considered general requirements on the curvature of an objective function to enable the capture of all Pareto points. Research continues on efficient methods to capture Pareto frontiers [16]. These researchers all address the problem of defining an objective function that may be used to locate all points on a Pareto frontier. In that optimization-oriented framework, the Pareto frontier is first uncovered and then the most desirable of the Pareto points is chosen. The research presented in this paper takes a different point of view, in which the parameters of a decision function are selected to pinpoint the most desirable option without the intermediate step of uncovering an entire Pareto frontier. The parameters that define the decision also determine which Pareto points are accessible. It is shown here that a known family of preference aggregation operators for design are a priori capable of accessing all Pareto points, when many other methods are not. It also gives an interpretation of the meaning of the parameters of the operator, a matter that is not addressed in the optimization literature except insofar as these parameters affect the ability of the function to capture Pareto points.

Weighted-sum formulations remain quite common in engineering design applications [17-20]. Recent research also includes alternative approaches to multicriteria optimization: Wassenaar and Chen [21] and Wassenaar et al. [22] advocate using the single criterion of consumer demand; Wan and Krishnamurty [23] use an iterative interactive procedure to avoid inconsistencies in the specification of utility functions, an approach that could be coupled with the techniques presented here; and Tappeta and Renaud [24] also focus on iterative discovery of preferences, in this case through repeated approximation of the local Pareto frontier. Farhang-Mehr and Azarm [25] discuss quality metrics to assess the "goodness" of Pareto points found by evolutionary algorithms, and Maddulapalli et al. discuss gradient methods [26]. Olewnik et al. [27] examine the relevance of Pareto optimality to so-called flexible systems. Wood and Agogino [28] propose a normative method for conceptual design that combines a heterogeneous design space model with expected value decision-making and information value theory. Franssen and Bucciarelli [29] argue that rationality in group decision making can be defined in gametheoretic terms considering the utilities of multiple decision makers.

Clearly, a weighted-sum approach to multicriteria decision making is problematic if it cannot identify all possible best solutions. In the example presented here, the performance calculations are all analytic expressions, which are easily evaluated, and the 
Pareto frontier is thus easily discovered. In such simple cases, a designer can choose to informally explore regions of the performance space that the formal decision model does not identify. When the design is more complicated, perhaps because evaluation is more costly (say, each point is a finite element calculation rather than an analytic expression) or because there are more than two competing objectives, informal exploration becomes much more difficult and designers may rely more on automated techniques, such as optimization. In these more complicated design situations, it is particularly important that design decision methods provide reliable guidance.

In the example presented above, it is clear that the choice of a point on the Pareto frontier depends on the trade-off between safety and mass. The example shows that a weighted-sum aggregation cannot model all possible trade-offs, and may miss large desirable regions of a performance space. It will be shown later that the difficulty highlighted by this example is not peculiar to a weighted sum. Intuitively it might seem possible to (continously) navigate a Pareto frontier from one end to the other by (continuously) varying the importance weights for two attributes. In fact, as is suggested by the problems exhibited here by the weighted sum, a formal model of a design decision requires, in addition, independent control over the degree of compensation among criteria. The full Pareto frontier cannot be identified by simply choosing importance weights alone, and even when it can be so identified, the choice of one point from that frontier depends on the degree of compensation as well as the importance weights.

\section{Aggregation Operators for Engineering Design}

All support methods for multicriteria decision making ultimately rely on the aggregation of disparate preferences with aggregation functions. In the MoI, for instance, customers and designers assign preference values between 0 (absolutely unacceptable) and 1 (most preferred) to various attributes. Mathematically, preferences are maps or functions from a set of design or performance variables to the closed unit interval $[0,1]$. Preference functions are denoted $\mu$, while the Greek letters $\alpha, \beta, \ldots$, will be used to indicate the values in $[0,1]$ that preferences may assume. The functions $\mu_{\text {mass }}$ and $\mu_{\text {safety }}$ in the example presented above are both preferences.

When making a decision based on preferences for two or more attributes, it is necessary to combine the separate preferences into a single preference map. A rigorous treatment of aggregation requires some mathematics concerning the distinction between aggregation operators and aggregation functions; these details are presented in the Appendix. For most purposes, an aggregation is a function $\mathcal{P}$, which takes two or more preference values $\left(\alpha_{1}, \alpha_{2}\right.$, etc), together with their importance weights, which are denoted $\omega_{i}$ and treated as parameters, and returns a single preference value

$$
\mu_{\mathrm{agg}}(\mathbf{x})=\mathcal{P}\left(\alpha_{1}, \cdots, \alpha_{n} ; \omega_{1}, \cdots, \omega_{n}\right)
$$

where $\mu_{i}(\mathbf{x})=\alpha_{i}$ for $i$ ranging over the $n$ attributes. In the example presented above, $\alpha_{1}$ was the preference for mass, $\alpha_{2}$ was the preference for safety, and the aggregation $\mathcal{P}$ was a weighted sum

$$
\mathcal{P}\left(\alpha_{1}, \alpha_{2} ; \omega_{1}, \omega_{2}\right)=\frac{\omega_{1} \alpha_{1}+\omega_{2} \alpha_{2}}{\omega_{1}+\omega_{2}}
$$

The aggregation of preference is defined for an arbitrary (finite) number of individual preference functions. The following discussion and results consider the case of aggregation of exactly two individual preferences. Aggregation of more than two preferences is accomplished using a pairwise hierarchy [30]. A structural automotive design example with hierarchical preferences was presented by Scott and Antonsson [31] using a reduced set of aggregation operators; that example was exercised with the full family of aggregation operators in Chapter 8 of Scott [9]. A more detailed discussion of the issues surrounding pairwise hierarchical aggregation is outside the scope of this paper and is planned for future
Table 2 Axioms of the Mol for aggregation functions

\begin{tabular}{ll}
\hline \hline AF.1 & Monotonicity \\
& $\mathcal{P}\left(\alpha_{1}, \alpha_{2} ; \omega_{1}, \omega_{2}\right) \leqslant \mathcal{P}\left(\alpha_{1}, \alpha_{2}^{\prime} ; \omega_{1}, \omega_{2}\right) \forall \alpha_{2} \leqslant \alpha_{2}^{\prime}$ \\
& $\mathcal{P}\left(\alpha_{1}, \alpha_{2} ; \omega_{1}, \omega_{2}\right) \leqslant \mathcal{P}\left(\alpha_{1}, \alpha_{2} ; \omega_{1}, \omega_{2}^{\prime}\right) \forall \omega_{2} \leqslant \omega_{2}^{\prime} ; \alpha_{1}<\alpha_{2}$ \\
& Symmetry \\
& $\mathcal{P}\left(\alpha_{1}, \alpha_{2} ; \omega_{1}, \omega_{2}\right)=\mathcal{P}\left(\alpha_{2}, \alpha_{1} ; \omega_{2}, \omega_{1}\right)$ \\
& Continuity \\
& $\mathcal{P}\left(\alpha_{1}, \alpha_{2} ; \omega_{1}, \omega_{2}\right)=\lim _{\alpha_{2}^{\prime} \rightarrow \alpha_{2}} \mathcal{P}\left(\alpha_{1}, \alpha_{2}^{\prime} ; \omega_{1}, \omega_{2}\right)$ \\
& $\mathcal{P}\left(\alpha_{1}, \alpha_{2} ; \omega_{1}, \omega_{2}\right)=\lim _{\omega_{2}^{\prime} \rightarrow \omega_{2}} \mathcal{P}\left(\alpha_{1}, \alpha_{2} ; \omega_{1}, \omega_{2}^{\prime}\right)$ \\
& Idempotency \\
AF.4 & $\mathcal{P}\left(\alpha, \alpha ; \omega_{1}, \omega_{2}\right)=\alpha \forall \omega_{1}+\omega_{2}>0$ \\
& Annihilation \\
AF.5 & $\mathcal{P}\left(\alpha, 0 ; \omega_{1}, \omega_{2}\right)=0 \forall \omega_{2} \neq 0$ \\
& Self-scaling weights \\
AF.6 & $\mathcal{P}\left(\alpha_{1}, \alpha_{2} ; \omega_{1} t, \omega_{2} t\right)=\mathcal{P}\left(\alpha_{1}, \alpha_{2} ; \omega_{1}, \omega_{2}\right) \forall \omega_{1}+\omega_{2}, t>0$ \\
& Zero weights \\
& $\mathcal{P}\left(\alpha_{1}, \alpha_{2} ; \omega_{1}, 0\right)=\alpha_{1} \forall \omega_{1} \neq 0$ \\
\hline \hline
\end{tabular}

publications. Note that in pairwise hierarchical aggregation, both importance weights and degree of compensation may be different for each pairwise comparison.

From a purely formal point of view, any operator could be used. Not all operators, however, are appropriate for decision making in engineering design. The class of possible operators can be restricted initially by supposing an intuitively reasonable set of axioms for engineering design preference aggregation. The axioms shown in Table 2 have been presented and discussed in detail previously [32], and aggregation functions, which fulfill these axioms, will be called design appropriate. Here we point out only axiom AF.4, idempotency, and axiom AF.5, annihilation, which distinguish the MoI from other decision methods. Multiattribute utility theory [33] is an alternative axiomatic approach to decision making and has been compared previously in some detail to the preference-aggregation approach presented here [9,34,35].

Scott and Antonsson [12] showed that the operators that satisfy the axioms in Table 2 are a restricted set of weighted means and that, in particular, there is a family of aggregation operators $\mathcal{P}_{S}$ that spans an entire range of possible operators between min and max, given by

$$
\mathcal{P}_{s}\left(\alpha_{1}, \alpha_{2} ; \omega_{1}, \omega_{2}\right)=\left(\frac{\omega_{1} \alpha_{1}^{s}+\omega_{2} \alpha_{2}^{s}}{\omega_{1}+\omega_{2}}\right)^{1 / s}
$$

It is readily shown [12] that

$$
\begin{gathered}
\mathcal{P}_{-\infty}=\lim _{s \rightarrow-\infty} \mathcal{P}_{s}=\min \\
\mathcal{P}_{0}=\lim _{s \rightarrow 0} \mathcal{P}_{s}=\left(\alpha_{1}^{\omega_{1}} \alpha_{2}^{\omega_{2}}\right)^{1 /\left(\omega_{1}+\omega_{2}\right)} \\
\mathcal{P}_{1}=\lim _{s \rightarrow 1} \mathcal{P}_{s}=\frac{\omega_{1} \alpha_{1}+\omega_{2} \alpha_{2}}{\omega_{1}+\omega_{2}} \\
\mathcal{P}_{\infty}=\lim _{s \rightarrow+\infty} \mathcal{P}_{s}=\max
\end{gathered}
$$

Note that $\mathcal{P}_{0}$ is the geometric mean, and $\mathcal{P}_{1}$ is the arithmetic mean or weighted sum. As noted above, both the degree of compensation $s$ and the ratio of importance weights may vary between comparison pairs in a hierarchical aggregation of more than two attributes. The implications and uses of this family of operators will be discussed below.

\section{Compensation Strategies: How to Consider All Designs}

In Sec. 2 it was seen that a weighted sum cannot always identify all Pareto points for a design. This is just one instance of a 
Table 3 Overall preferences for three points with $s=1$ and $s$ $=2$

\begin{tabular}{ccccc}
\hline \hline point & $\alpha_{1}$ & $\alpha_{2}$ & $\mathcal{P}_{1}\left(\alpha_{1}, \alpha_{2} ; \omega_{1}, \omega_{2}\right)$ & $\mathcal{P}_{2}\left(\alpha_{1}, \alpha_{2} ; \omega_{1}, \omega_{2}\right)$ \\
\hline A (min mass) & 1 & 0 & 0.5 & 0.71 \\
B ("compromise") & 0.97 & 0.07 & 0.52 & 0.69 \\
C (max safety) & 0 & 1 & 0.5 & 0.71 \\
\hline \hline
\end{tabular}

more general result about preference aggregation functions: in fact, no fixed degree of compensation (e.g., a weighted sum) can always identify all Pareto points.

It was noted by Otto and Antonsson [11] that, in addition to importance weights, the degree of compensation among goals is a variable feature of multiattribute decision problems. In particular, decisions were classified as either compensating or noncompensating. A noncompensating solution was said to hold when the most desirable solution was the one that maximizes the performance of the lowest-performing attribute; this case was modeled mathematically by the minimum operator

$$
\mu_{\text {agg }}(\mathbf{x})=\min \left(\alpha_{1}, \alpha_{2}\right)
$$

where $\mu_{1}(\mathbf{x})=\alpha_{1}, \mu_{2}(\mathbf{x})=\alpha_{2}$. In compensating decisions, higher performance on one attribute could partially make up for lower performance on another attribute; this situation was modeled using a weighted product of powers (also called the weighted geometric mean):

$$
\mu_{\mathrm{agg}}(\mathbf{x})=\left(\alpha_{1}{ }^{\omega_{1}} \alpha_{2}{ }^{\omega_{2}}\right)^{1 /\left(\omega_{1}+\omega_{2}\right)}
$$

The family of aggregation operators $\mathcal{P}_{s}$ presented by Scott and Antonsson [12] makes clear that compensation is not an either/or proposition, and indeed that there is a continuous spectrum of compensation degrees between $\min \left(\right.$ at $\mathcal{P}_{-\infty}$ ) and $\max \left(\right.$ at $\mathcal{P}_{+\infty}$ ). As with importance weights, there is an intuitive notion that by continuously varying the degree of compensation, it should be possible to navigate a Pareto frontier; the exact significance of the "compensation parameter" $s$, however, is not immediately obvious. The meaning of the parameter $s$ as a measure of degree of compensation can be made more precise in two ways. First, the overall preference for any particular alternative increases with $s$ :

Proposition 2. $\mathcal{P}_{s}\left(\alpha_{1}, \alpha_{2} ; \omega_{1}, \omega_{2}\right)$ is nondecreasing as a function of $s$

$$
\frac{d}{d s} \mathcal{P}_{s}\left(\alpha_{1}, \alpha_{2} ; \omega_{1}, \omega_{2}\right) \geqslant 0
$$

All proofs are presented in the Appendix. Dyckhoff and Pedrycz [36] prove a result similar to Proposition 2.

To relate Proposition 2 to the example presented above, consider the three "optimal" points shown in Fig. 3. Using the weighted sum $(s=1)$ and equal importance weights $\left(\omega_{1}=\omega_{2}\right)$, the overall preferences for these three points are as shown in the penultimate column in Table 3. Proposition 2 states that if a larger value of $s$ is used, then the overall preference for all points will be larger. For instance, setting $s=2$ generates the final column of Table 3. If a value of $s<1$ were used, overall preference would decrease for all points.

The data in Table 3 show that the absolute overall preference for a particular point is not a meaningful measure of the degree of compensation. In comparing alternatives, it is the order of the relative preferences, not the absolute value of those preferences, that matters. Note that solution B was considered best of the three for $s=1$, but that both $\mathrm{A}$ and $\mathrm{C}$ were found to be superior to $\mathrm{B}$ when $s=2$.

The second measure of degree of compensation indicates which sorts of designs (relatively compensating or noncompensating) are favored by the aggregation. Consider any two alternatives $\mathrm{A}$ and $\mathrm{B}$, with preferences $\mu(\mathrm{A})=\left(\alpha_{1}, \alpha_{2}\right), \mu(\mathrm{B})=\left(\beta_{1}, \beta_{2}\right)$, and with $\alpha_{1}$ $>\beta_{1}, \beta_{2}>\alpha_{2}$. The alternatives $\mathrm{A}$ and $\mathrm{B}$ in the tables above, for instance, fulfill this condition. In this case, the least compensating function, the $\min (s=-\infty)$, dictates a choice of B over A (regardless of weights, provided they are nonzero). The most compensating function, the $\max (s=+\infty)$, dictates that A is preferred to B. Proposition 2 states only that the overall preference for A and the overall preference for B both increase as the parameter $s$ increases. The second interpretation of the degree of compensation shows that the relative preference for $\mathrm{A}$ (the option selected by the most compensating function) over B (the option selected by the least compensating function) increases with the compensation parameter. This measure shows that increasing $s$ tends to favor $\left(\alpha_{1}, \alpha_{2}\right)$ over $\left(\beta_{1}, \beta_{2}\right)$, in that the ratio $\mathcal{P}_{s}(\alpha) / \mathcal{P}_{s}(\beta)$ is an increasing function of $s$.

Proposition 3. If $\left(\alpha_{1}, \alpha_{2}\right),\left(\beta_{1}, \beta_{2}\right)$ are two preference points, with $\alpha_{1}>\beta_{1}, \beta_{2}>\alpha_{2}$, then

$$
\frac{d}{d s} \frac{\mathcal{P}_{s}\left(\alpha_{1}, \alpha_{2} ; \omega_{1}, \omega_{2}\right)}{\mathcal{P}_{s}\left(\beta_{1}, \beta_{2} ; \omega_{1}, \omega_{2}\right)} \geqslant 0
$$

as long as $\omega_{1}, \omega_{2}>0$.

Proposition 3 states that as the parameter $s$ increases, the relative preference for A rather than B increases as well. In this case, there will be a value $s^{*}$ at which the preferences for A and B will be identical; for $s<s^{*}$, B will be preferred to A, while for $s>s^{*}$, A will be preferred to $\mathrm{B}$. Thus the parameter $s$ is indeed a measure of degree of compensation among goals. For the A and B discussed above, $s^{*}=1.239$; while the importance weights remain equal, strategies with $s>1.239$ (more compensating than $\mathcal{P}_{1.239}$ ) select A over B, strategies with $s<1.239$ (less compensating than $\left.\mathcal{P}_{1.239}\right)$ select B over A.

\section{Selecting Among Pareto Optimal Points}

Two different ways of quantifying trade-offs between preferences have now been discussed. One is the use of numerical weights to quantify the relative importance of different attributes; it was seen that these importance weights alone do not suffice to capture all reasonable decisions. The second is the assessment of a compensation parameter ( $s$ in Eq. (5)) to quantify the degree of compensation between two attributes. In this section, it is shown that an aggregation of two preferences actually depends on both these parameters: the compensation parameter $s$, and the ratio of the weights $\omega_{1}$ and $\omega_{2}$ assigned to the two preferences. (It follows from axiom AF.6 that only the ratio of weights is significant.) By appropriately selecting both parameters it is possible to model any "reasonable" decision. Furthermore, both degree of compensation and weights must be considered to capture all potentially acceptable decisions.

The key result about the parameterized family of functions defined in (5) is that, unlike a weighted sum, it can be used to select any undominated point. If both degree of compensation and weighting can be considered, then it is possible to model a decision to choose any Pareto optimal solution. That is, for any individual in a set of undominated solutions, there exist a degree of compensation $s$ and a ratio of weights $\omega=\omega_{2} / \omega_{1}$ that select that individual as the "best" overall solution:

Proposition 4. For any pair $\left(\alpha_{1}, \alpha_{2}\right)$ in an undominated finite set $M$ of preference pairs, there exist $s^{*}$ and $\omega^{*}=\omega_{2}{ }^{*} / \omega_{1}{ }^{*}$ such that

$$
\mathcal{P}_{s^{*}}\left(\alpha_{1}, \alpha_{2} ; 1, \omega^{*}\right)=\max _{\left(\alpha_{i}, \alpha_{j}\right) \in M} \mathcal{P}_{s^{*}}\left(\alpha_{i}, \alpha_{j} ; 1, \omega^{*}\right)
$$

Proposition 4 says that it is mathematically possible to choose a ratio of weights and a degree of compensation to select any particular undominated point, at least from a finite set of possible solutions. Consider the Pareto frontier calculated for the truss example, paying special attention to three points labeled $\mathrm{A}, \mathrm{B}$, and $\mathrm{C}$ (see Fig. 5 and Table 4).

Recall that point $\mathrm{B}$ could not be selected by the standard weighted-sum approach $(s=1)$, for any choice of weights $\omega_{1}, \omega_{2}$. 


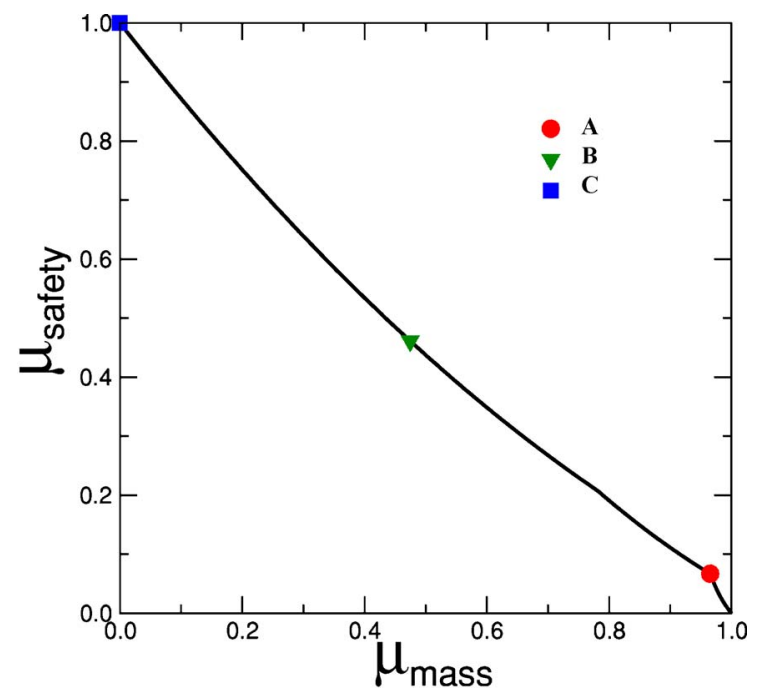

Fig. 5 Pareto frontier with selected points A, B, and C

A strategy that uses the degree of compensation $s=-1$, however, chooses B over all other Pareto points when the importance weights are equal. For any other point on the Pareto frontier, there is some choice of $s$ and $\omega$ that selects that point above all the others. A method to simultaneously determine a degree of compensation $s$ and a weight ratio $\omega$ for a given problem was presented by Scott and Antonsson [37].

The special case of only two (undominated) candidates is a case that can be resolved using only importance weights. Even with a fixed degree of compensation, by a judicious selection of weights, it is always possible to select one of two undominated points:

Proposition 5. If $\left(\alpha_{1}, \alpha_{2}\right)$ and $\left(\beta_{1}, \beta_{2}\right)$ are two preference points, neither of which dominates the other (assume without loss of generality that $\alpha_{1}>\beta_{1}$ and $\beta_{2}>\alpha_{2}$ ), then for every degree of compensation $s \in(-\infty,+\infty)$, there is a weight $\omega$ such that

$$
\mathcal{P}_{s}\left(\alpha_{1}, \alpha_{2} ; 1, \omega\right)=\mathcal{P}_{s}\left(\beta_{1}, \beta_{2} ; 1, \omega\right)
$$

However, the ability to choose either one of two undominated points does not imply the ability to choose even any one of three dominated points. Consider the example of the three alternatives $\mathrm{A}, \mathrm{B}$, and $\mathrm{C}$ discussed immediately above. The preferences are

$$
\begin{gathered}
\left(\alpha_{1}, \alpha_{2}\right)=\left[\mu_{1}(\mathrm{~A}), \mu_{2}(\mathrm{~A})\right]=(0.97,0.07) \\
\left(\beta_{1}, \beta_{2}\right)=\left[\mu_{1}(\mathrm{~B}), \mu_{2}(\mathrm{~B})\right]=(0.47,0.46) \\
\left(\gamma_{1}, \gamma_{2}\right)=\left[\mu_{1}(\mathrm{C}), \mu_{2}(\mathrm{C})\right]=(0,1)
\end{gathered}
$$

which are mutually undominated. Using a weighted sum with its fixed degree of compensation $(s=1)$, let the weight assigned to the first preference of the ordered pair be fixed at 1 and let $\omega$ be the weight associated with the second preference. Then if $\omega<1.28, \alpha$ is preferred to $\beta$. If $\omega>0.87, \gamma$ is preferred to $\beta$. Thus there is no weighting that can select $\beta$ over both $\gamma$ and $\alpha$; this undominated point will never be selected as long as $s=1$. (It was shown above that this point is easily selected when $s=-1$.) This problem is not unique to the weighted sum, but is present for all aggregation methods that dictate a single fixed degree of compensation between attributes.

Proposition 6. For any fixed degree of compensation $s \neq-\infty$, for any preference point $\left(\alpha_{1}, \alpha_{2}\right)$ with $0<\alpha_{1}, \alpha_{2}<1$, there exist $\left(\beta_{1}, \beta_{2}\right),\left(\gamma_{1}, \gamma_{2}\right)$, such that all three points are mutually undominated but

$$
\mathcal{P}_{s}\left(\alpha_{1}, \alpha_{2} ; 1, \omega\right)<\mathcal{P}_{s}\left(\beta_{1}, \beta_{2} ; 1, \omega\right)
$$

or

$$
\mathcal{P}_{s}\left(\alpha_{1}, \alpha_{2} ; 1, \omega\right)<\mathcal{P}_{s}\left(\gamma_{1}, \gamma_{2} ; 1, \omega\right)
$$

for all possible weights $\omega$.

Of course, it is possible to choose a degree of compensation and a weight sequentially, rather than simultaneously, and achieve any undominated point. Any method, however, that restricts aggregation a priori to a single fixed degree of compensation potentially excludes some undominated points from consideration.

Similarly, when weights are determined first, some undominated points may become unattainable by any choice of degree of compensation. Consider this example: if weights are unrestricted, then the two preference points $\alpha=(0.5,0.9)$ and $\beta=(0.6,0.1)$ do not dominate each other, and there exist compensation-weight pairs to select either one over the other. If the weights are declared equal, then the order of elements is irrelevant and $\alpha$ dominates $\beta$. Indeed, whenever the second element has a higher weight than the first, then $\alpha$ dominates $\beta$. This situation only arises, however, when reversing the order of one preference makes one preference dominate the other. Whenever two points do not dominate each other, even if the elements of one point are reversed, then for any weight there is a degree of compensation that will achieve equality. When reversing the order of elements allows one point to dominate the other, then there are always some weights that preclude equality for all degrees of compensation.

Proposition 7. If $\left(\alpha_{1}, \alpha_{2}\right)$ and $\left(\beta_{1}, \beta_{2}\right)$ are two preference points, neither of which dominates the other (assume without loss of generality that $\alpha_{1}>\beta_{1}$ and $\beta_{2}>\alpha_{2}$ ), then the following are true:

1. If $\alpha_{1}>\beta_{1}, \beta_{2}>\alpha_{2}$, then for all $\omega>0$ there exists a degree of compensation s such that $\mathcal{P}_{s}\left(\alpha_{1}, \alpha_{2} ; 1, \omega\right)=\mathcal{P}_{s}\left(\beta_{1}, \beta_{2} ; 1, \omega\right)$.

2. If $\alpha_{1}>\beta_{2}>\alpha_{2}>\beta_{1}$, then there exists a weight $\omega>0$ such that there is no degree of compensation $s$ such that $\mathcal{P}_{s}\left(\alpha_{1}, \alpha_{2} ; 1, \omega\right)=\mathcal{P}_{s}\left(\beta_{1}, \beta_{2} ; 1, \omega\right)$.

In summary, if the degree of compensation $s$ is chosen in advance and is not the $\min (s=-\infty)$ or the $\max (s=+\infty)$, then it is always possible to choose one point of two undominated points by appropriate choice of weights. However, it is no longer always possible to choose any one point from an undominated set. Methods which rely exclusively on the arithmetic mean, or on any other fixed degree of compensation, do not truly consider all undominated points as potential solutions. Neither is a choice of a degree of compensation by itself enough to choose among any set of undominated points. In order for a decision model to consider all possible decisions it is necessary and sufficient that both degree of compensation and importance weights be appropriately established.

Table 4 Performances and preferences of three points from Fig. 5

\begin{tabular}{ccccccc}
\hline \hline Point & Mass & Safety & $\alpha_{1}$ & $\alpha_{2}$ & $\mathcal{P}_{1}\left(\alpha_{1}, \alpha_{2} ; 1,1\right)$ & $\mathcal{P}_{-1}\left(\alpha_{1}, \alpha_{2} ; 1,1\right)$ \\
\hline A & 129 & 1.6 & 0.97 & 0.07 & 0.52 & 0.13 \\
B & 217 & 5.15 & 0.47 & 0.46 & 0.47 & 0.46 \\
C & 302 & 10 & 0 & 1 & 0.5 & 0 \\
\hline \hline
\end{tabular}




\section{Example}

Returning to the example of the bracket shown in Fig. 1, recall that the bracket is made of aluminum (6061-T6), which has the following relevant material properties:

Young's modulus $(E) \quad 69 \times 10^{9} \mathrm{~Pa}$

Density $(\rho) \quad 2660 \mathrm{~kg} / \mathrm{m}^{3}$

Yield stress $(\sigma) \quad 275 \times 10^{6} \mathrm{~Pa}$

Recall as well that there are four design variables:

$x \in[0.1 \mathrm{~m}, 0.9 \mathrm{~m}]$ distance from wall to pin

$t \in[5 \mathrm{~mm}, 20 \mathrm{~mm}]$ thickness of bending member

$h \in[5 \mathrm{~mm}, 20 \mathrm{~mm}]$ height of bending member

$w \in[5 \mathrm{~mm}, 20 \mathrm{~mm}]$ width of (square) compression member

as well as two fixed parameters:

$P=1 \mathrm{~kg}$ load supported by structure

$L=1 \mathrm{~m}$ distance from supported load to wall

The first performance measure is total mass $M$ (in $\mathrm{kg}$ )

$$
M=\rho\left(h t L+w^{2} \sqrt{x^{2}+y^{2}}\right)=2660\left(h t+w^{2} \sqrt{x^{2}+0.25}\right)
$$

The safety factor $S$ has two components, the safety factor for the bending member $S_{b}$ and the safety factor for the compression member $S_{c}$. Since the yield stress in the bending member is $\sigma$, and the maximum stress in the bending member is $12 P(L-x) / t h^{2}$, the factor of safety in bending is the ratio

$$
S_{b}=\frac{\sigma t h^{2}}{12 P(L-x)}=\frac{\sigma t h^{2}}{120(1-x)}
$$

Similarly, using the Euler buckling load, the safety factor in the compression member is

$$
S_{c}=\frac{\pi^{2} E x y w^{4}}{12 P L\left(x^{2}+y^{2}\right)^{1.5}}=\frac{\pi^{2} E x y w^{4}}{120\left(x^{2}+0.25\right)^{1.5}}
$$

The safety factor for the entire design is defined to be the minimum of the two

$$
\mathrm{S}=\min \left(\frac{\sigma t h^{2}}{120(1-x)}, \frac{\pi^{2} \mathrm{E} x y w^{4}}{120\left(x^{2}+0.25\right)^{1.5}}\right)
$$

Note that this is a strictly noncompensating aggregation $(s=-\infty)$ of the two individual safety factors.

The design problem is to minimize the mass while maximizing the factor of safety; both are analytic expressions. First, note that mass is linear in both $t$ and $h$, whereas the factor of safety in bending is linear in $t$ but quadratic in $h$. Thus, as long as no other design variables reach the maximum acceptable dimensions, it will always be preferable to increase $h$ rather than $t$. Setting $t$ $=t_{\min }=5 \mathrm{~mm}$ reduces the problem to the three design variables $x, h$, and $w$. Both $h$ and $w$ can be expressed as functions of $x$ and a safety factor, and thus finding the minimum possible mass for a given safety factor requires solving a rational equation in $x$. These solutions yield a Pareto frontier of designs, which is shown in Fig. 6. Note that in Figs. 6 and 7 the Pareto frontier is plotted in performance space (i.e., safety factor versus mass). In all other graphs the preferences for the performances are plotted, which is why the Pareto frontier curve appears backward here. The change is made for these two figures so the design variable values that correspond to the best performances may be presented.

The values of $x, h$, and $w$, which generate these optimal designs, are included in Fig. 7. It can be seen from Fig. 7 that at each Pareto point, at least one domain constraint is active: in particular, for low mass, $h$ takes its minimum acceptable value of $5 \mathrm{~mm}$, whereas for higher mass, $x$ takes its maximum acceptable value of $0.9 \mathrm{~m}$. Nevertheless, along most of the Pareto frontier two design variables are changing as the frontier is traversed.

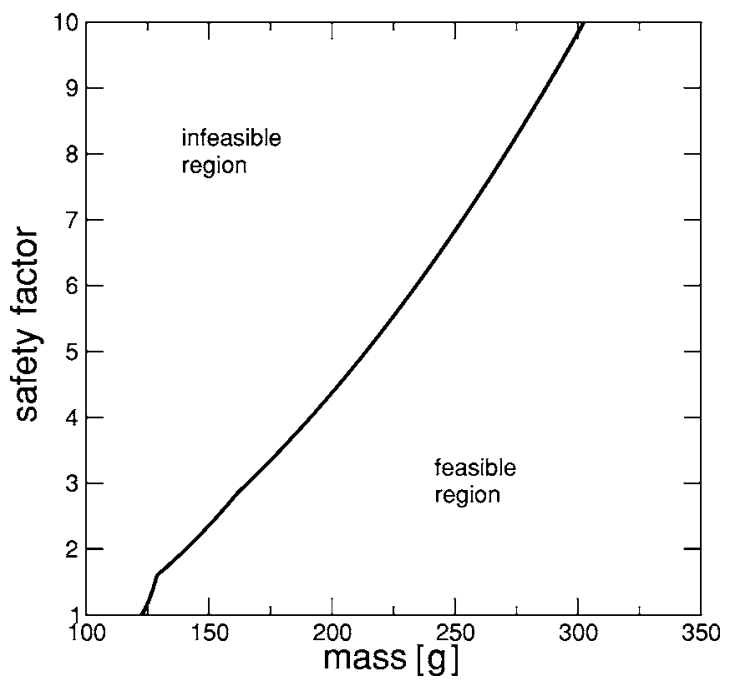

Fig. 6 Pareto frontier of best performances

Taking the minimum and maximum values on the Pareto frontier for weight and factor of safety, setting the best and worst values to preferences of $\mu=1$ and $\mu=0$, respectively, the Pareto frontier can be normalized as shown in Fig. 8; note that this preference-based display is the format that was used earlier. Further note that the utopia point is now the upper right-hand corner of the graph, since preference for mass decreases with mass. The three "optimal" points found earlier by the weighted-sum method are shown as black circles on the graph in Fig. 8.

By allowing both weights and degrees of compensation to vary, any of the points on the Pareto frontier may be selected as the highest performing point. For this example, setting $s=-1$ and varying the weights allows for a more varied range of "best" designs (see Table 5). These points are shown as squares on the graph in Fig. 8.

By allowing the weight assigned to one attribute to be much larger than the weight assigned to the other, points much closer to the extremes of the Pareto frontier can be reached with $s=-1$ (see Table 6). These points are shown as triangles on the graph in Fig. 8.

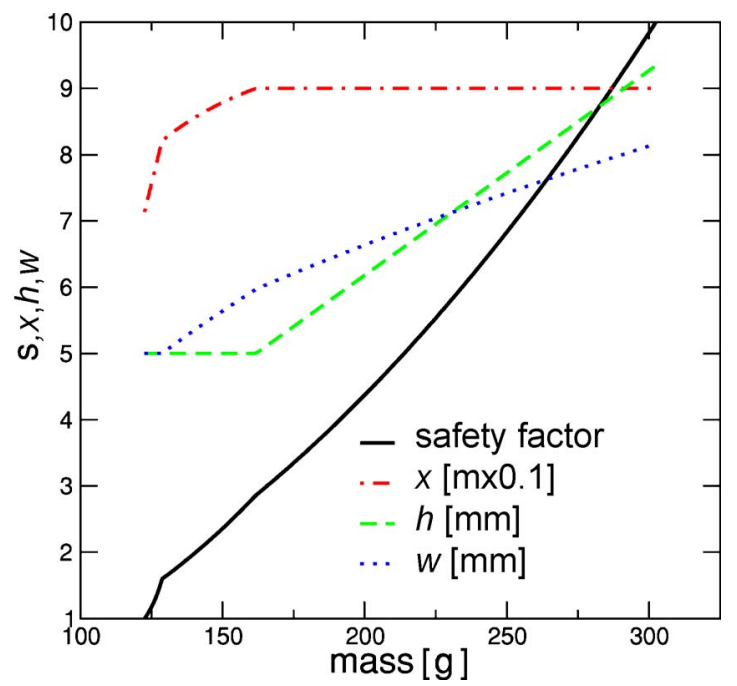

Fig. 7 Pareto frontier with design variable values 


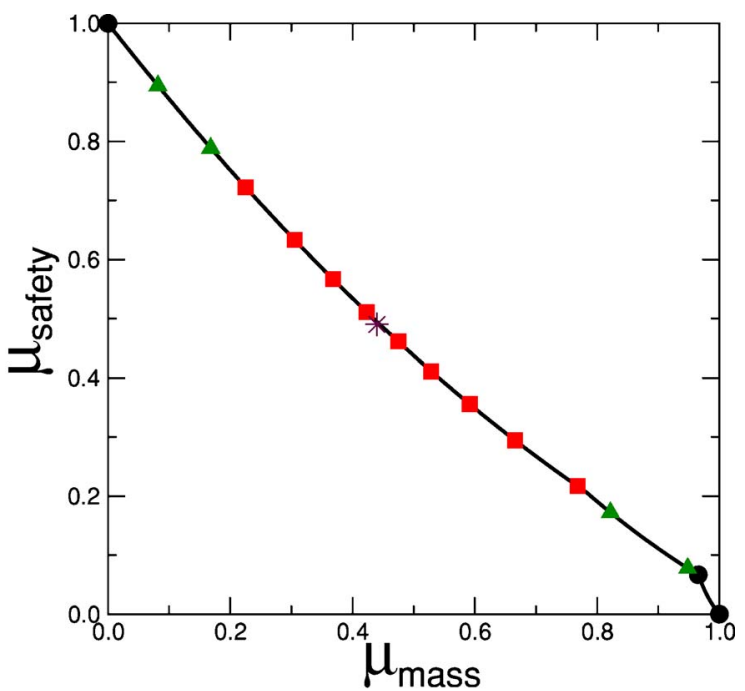

Fig. 8 Normalized Pareto frontier

\section{Conclusion}

Weighted-sum aggregation with importance weights is common to many methods for engineering design decision making. Three important difficulties with these methods are the inability of weighted-sum methods to select all Pareto points, the arbitrary nature of direct assignment of importance weights, and the fact that these methods have a fixed degree of compensation among criteria. Although the inability of weighted-sum methods to recover nonconvex Pareto frontiers is well known in the optimization literature, research into parametrized objective functions that can capture entire Pareto frontiers has not discussed the use of function parameters to define correct decisions. This paper has presented an interpretation of the decision parameters of a family of weighted means that has been developed previously for engineering design decision making.

It is shown here that a complete model of an engineering decision depends not only on the importance weights, but also on the degree of compensation. A weighted sum, or any other predetermined aggregation procedure, is overly and inappropriately constraining. The appropriate degree of compensation among attributes is situation dependent, and a "one-size-fits-all" decision method that dictates an aggregation method can lead to incorrect results. For the two-attribute case, an easily computed family of

Table 5 Optimal points for $s=-1$ and $\omega_{1} \in[0.1,0.9]$

\begin{tabular}{llllll}
\hline \hline$\omega_{1}$ & Weight & Safety & $x$ & $h$ & $w$ \\
\hline 0.1 & 2.62 & 7.5 & 0.9 & 8.09 & 7.59 \\
0.2 & 2.48 & 6.7 & 0.9 & 7.65 & 7.38 \\
0.3 & 2.36 & 6.1 & 0.9 & 7.30 & 7.21 \\
0.4 & 2.26 & 5.6 & 0.9 & 6.99 & 7.06 \\
0.5 & 2.17 & 5.15 & 0.9 & 6.70 & 6.91 \\
0.6 & 2.07 & 4.7 & 0.9 & 6.41 & 6.76 \\
0.7 & 1.96 & 4.2 & 0.9 & 6.05 & 6.57 \\
0.8 & 1.83 & 3.65 & 0.9 & 5.64 & 6.34 \\
0.9 & 1.64 & 2.95 & 0.9 & 5.07 & 6.01 \\
\hline \hline
\end{tabular}

Table 6 Optimal points for $s=-1$ and large weight ratios

\begin{tabular}{cccccc}
\hline \hline$\omega_{1}$ & weight & safety & $x$ & $h$ & $w$ \\
\hline 0.01 & 2.88 & 9.05 & 0.9 & 8.89 & 7.96 \\
0.05 & 2.72 & 8.1 & 0.9 & 8.41 & 7.74 \\
0.95 & 1.55 & 2.55 & 0.89 & 5 & 5.77 \\
0.99 & 1.32 & 1.7 & 0.83 & 5 & 5.10 \\
\hline \hline
\end{tabular}

preference-aggregation functions is completely determined by two parameters that represent the trade-off strategy (degree of compensation and ratio of importance weights). A number of results concerning aggregation functions were presented, formalizing the definition of the term "compensation" and proving that this parametrized family of aggregation functions, unlike methods that fix the degree of compensation, is capable of modeling all rational design decisions.

The correct choice of a level of compensation $s$ and importance weights $\omega_{i}$ for a particular decision should not be made arbitrarily, as the arbitrary specification of these values can lead to undesired results. A systematic method for determining the values of these parameters using a method of indifference points has been presented previously [37]. The research presented here provides mathematical and theoretical background underpinning that systematic method. It was seen that as the value of the compensation parameter $s$ increases, so does the propensity of the function to select designs with one high performance and one low performance over designs with average performance with respect to both attributes; this provides an interpretation of the term "compensation" in engineering design. A related result is that the choice of one member of a Pareto set by specifying importance weights is sensitive to the degree of compensation, showing that while a higher importance weight will tend to favor one attribute over another, there can be no absolute interpretation of the meaning of importance weights in the absence of the degree of compensation. Any method that intends to provide an absolute interpretation of importance weights by fixing the degree of compensation may miss some Pareto points. Thus the only methods that are capable of defining sensible decisions without running the risk of missing desired points are methods that can model a full range of both importance weights and degrees of compensation.

\section{Acknowledgments}

This material is based on work supported, in part, by the National Science Foundation under NSF Grant No DMI-981312I. Any opinions, findings, conclusions, or recommendations expressed in this publication are those of the authors and do not necessarily reflect the views of the sponsors.

\section{Appendix: Definitions and Proofs}

Definition 8. A preference is a map $\mu: \mathbf{X} \rightarrow[0,1]$, where $\mathbf{X}$ is a set, usually of design or performance variables.

Definition 9. For a set of preferences $\mu_{i}: \mathbf{X} \rightarrow[0,1]$, the aggregation of the $\mu_{i}$ is itself a preference $\mu_{\mathrm{agg}}: \mathbf{X} \rightarrow[0,1]$, defined (pointwise) by a functional aggregation operator $\mathcal{P}: P F[\mathbf{X}]^{n}$ $\times \mathrm{R}^{+n} \backslash\{0\} \rightarrow P F[\mathbf{X}]$

$$
\mu_{\text {agg }}(\mathbf{x})=\mathcal{P}\left(\mu_{1}, \ldots, \mu_{n} ; \omega_{1}, \ldots, \omega_{n}\right)(\mathbf{x})
$$

where the parameters $\omega_{i} \in \mathbb{R}^{+}$are weights, and $P F[\mathbf{X}]$ is the space of preference functions on $\mathbf{X}$, the set $\{\mu \mid \mu: \mathbf{X} \rightarrow[0,1]\}$. An aggregation operator must satisfy:

$$
\mathcal{P}\left(\mu_{1}, \cdots, \mu_{n} ; \omega_{1}, \cdots, \omega_{n}\right)\left(\mathbf{x}_{1}\right)=\mathcal{P}\left(\mu_{1}{ }^{\prime}, \cdots, \mu_{n}{ }^{\prime} ; \omega_{1}, \cdots, \omega_{n}\right)\left(\mathbf{x}_{2}\right)
$$

whenever $\mu_{i}\left(\mathbf{x}_{1}\right)=\mu_{i}^{\prime}\left(\mathbf{x}_{2}\right)$ for all $i$.

Following Definition 9 , a preference aggregation operator $\mathcal{P}$ takes as its inputs two preference functions $\mu_{1}$ and $\mu_{2}$, together with the weighting parameters $\omega_{1}$ and $\omega_{2}$, and returns an aggregated preference function $\mu_{\mathrm{agg}}$. It is also possible to consider the map $\hat{\mathcal{P}}:[0,1]^{2} \times \mathbb{R}^{+2} \backslash\{0\} \rightarrow[0,1]$, which operates directly on preference values, rather than the aggregation operator $\mathcal{P}$. If $\mu_{1}(\mathbf{x})$ $=\alpha_{1}$ and $\mu_{2}(\mathbf{x})=\alpha_{2}$ at some point $\mathbf{x}$, it may be desirable that there be a $\hat{\mathcal{P}}$ that allows the calculation of aggregated preference by composition 


$$
\mathcal{P}\left(\mu_{1}, \mu_{2} ; \omega_{1}, \omega_{2}\right)(\mathbf{x})=\hat{\mathcal{P}}\left[\mu_{1}(\mathbf{x}), \mu_{2}(\mathbf{x}) ; \omega_{1}, \omega_{2}\right]=\hat{\mathcal{P}}\left(\alpha_{1}, \alpha_{2} ; \omega_{1}, \omega_{2}\right)
$$

The requirement (26) that aggregated preferences be consistent for all $\mathbf{x}$ implies that $\hat{\mathcal{P}}$ is well defined, and indeed that $\mathcal{P}$ and $\hat{\mathcal{P}}$ can be identified with each other. Call $\hat{\mathcal{P}}$ the aggregation function corresponding to the aggregation operator $\mathcal{P}$. When it is clear from context, the ${ }^{\wedge}$ notation may be dropped and $\mathcal{P}$ will represent the aggregation function.

Proof of Proposition 2. Assume, for computational simplicity, that $\omega_{1}+\omega_{2}=1$. This assumption can be made without loss of generality by invoking axiom AF.6. Then

$$
\mathcal{P}_{s}\left(\alpha_{1}, \alpha_{2} ; \omega_{1}, \omega_{2}\right)=\left(\omega_{1} \alpha_{1}^{s}+\omega_{2} \alpha_{2}^{s}\right)^{1 / s}
$$

and

$$
\begin{aligned}
\frac{d}{d s} \mathcal{P}_{s}\left(\alpha_{1}, \alpha_{2} ; \omega_{1}, \omega_{2}\right)= & \frac{1}{s} \mathcal{P}_{s}\left(\alpha_{1}, \alpha_{2} ; \omega_{1}, \omega_{2}\right)\left[\left(\omega_{1} \alpha_{1}{ }^{s}\right.\right. \\
& \left.+\omega_{2} \alpha_{2}{ }^{s}\right)^{-1}\left(\omega_{1} \alpha_{1}{ }^{s} \log \alpha_{1}+\omega_{2} \alpha_{2}{ }^{s} \log \alpha_{2}\right) \\
& \left.-\frac{1}{s} \log \left(\omega_{1} \alpha_{1}{ }^{s}+\omega_{2} \alpha_{2}{ }^{s}\right)\right]
\end{aligned}
$$

In the case that $s<0$, we wish to establish that

$$
\begin{aligned}
& \left(\omega_{1} \alpha_{1}{ }^{s}+\omega_{2} \alpha_{2}{ }^{s}\right)^{-1}\left(\omega_{1} \alpha_{1}{ }^{s} \log \alpha_{1}+\omega_{2} \alpha_{2}{ }^{s} \log \alpha_{2}\right)-\frac{1}{s} \log \left(\omega_{1} \alpha_{1}{ }^{s}\right. \\
& \left.+\omega_{2} \alpha_{2}{ }^{s}\right) \leqslant 0
\end{aligned}
$$

Multiplying both sides by $s\left(\omega_{1} \alpha_{1}{ }^{s}+\omega_{2} \alpha_{2}{ }^{s}\right)$, which is nonpositive, we now require

$$
\begin{aligned}
s\left(\omega_{1} \alpha_{1}{ }^{s} \log \alpha_{1}+\omega_{2} \alpha_{2}{ }^{s} \log \alpha_{2}\right) \geqslant & \left(\omega_{1} \alpha_{1}^{s}+\omega_{2} \alpha_{2}^{s}\right) \log \left(\omega_{1} \alpha_{1}^{s}\right. \\
& \left.+\omega_{2} \alpha_{2}^{s}\right)
\end{aligned}
$$

Bringing $s$ inside on the left side of the inequality, we now need to show

$$
\begin{aligned}
\omega_{1} \alpha_{1}^{s} \log \left(\alpha_{1}{ }^{s}\right)+\omega_{2} \alpha_{2}{ }^{s} \log \left(\alpha_{2}{ }^{s}\right) \geqslant & \left(\omega_{1} \alpha_{1}{ }^{s}+\omega_{2} \alpha_{2}{ }^{s}\right) \log \left(\omega_{1} \alpha_{1}{ }^{s}\right. \\
& \left.+\omega_{2} \alpha_{2}{ }^{s}\right)
\end{aligned}
$$

Since $x \log x$ is a concave function for $x>0$, as $\left(d^{2} / d x^{s}\right) x \log x$
$=(1 / x)>0$, this is the case. Let $g(x)=x \log x, x_{1}=\alpha_{1}^{s}, x_{2}=\alpha_{2}^{s}$. Then

$$
g\left(\omega_{1} x_{1}+\omega_{2} x_{2}\right) \leqslant \omega_{1} g\left(x_{1}\right)+\omega_{2} g\left(x_{2}\right)
$$

proving (32).

If $s>0$, the inequality must be reversed in (30), but since $\left(\omega_{1} \alpha_{1}^{s}+\omega_{2} \alpha_{2}^{s}\right)$ is now non-negative, the sign of the inequality is recovered in (31).

This proves the proposition. It should be noted that if $\alpha_{1}$ and $\alpha_{2}$ are nonzero, and not equal, then strict inequalities hold throughout the proof.

The proof of Proposition 3 requires a lemma:

Lemma 10. Let

$$
h(x, y ; v, w)=\frac{v x \log (x)+w y \log (y)-(v x+w y) \log (v x+w y)}{v x+w y}
$$

If $x, y, v, w>0$, then

$$
\frac{d}{d x} h(x, y ; v, w)\left\{\begin{array}{lll}
<0 & \text { if } & x<y \\
>0 & \text { if } & x>y
\end{array}\right.
$$

Proof of Lemma 10.

$$
\frac{d}{d x} h(x, y ; v, w)=\frac{v w y \log (x / y)}{(v x+w y)^{2}}
$$

Since all other terms are positive, the sign depends on the sign of $\log (x / y)$, which is indeed positive for $x>y$ and negative for $x<y$.

It is a consequence of Lemma 10 that if the interval $\left(x_{1}, x_{2}\right)$ is wholly contained inside the interval $\left(y_{1}, y_{2}\right)$, then $h\left(x_{1}, x_{2} ; v, w\right)$ $<h\left(y_{1}, y_{2} ; v, w\right)$.

Proof of Proposition 3. With a little algebra, (d/ $d s)\left[\mathcal{P}_{s}\left(\alpha_{1}, \alpha_{2} ; \omega_{1}, \omega_{2}\right) / \mathcal{P}_{s}\left(\beta_{1}, \beta_{2} ; \omega_{1}, \omega_{2}\right)\right]$ is seen to be

$$
\frac{\mathcal{P}_{s}\left(\alpha_{1}, \alpha_{2} ; \omega_{1}, \omega_{2}\right)}{s^{2} \mathcal{P}_{s}\left(\beta_{1}, \beta_{2} ; \omega_{1}, \omega_{2}\right)}(Y-Z)
$$

where

$$
Y=\frac{\omega_{1} \alpha_{1}{ }^{s} \log \left(\alpha_{1}{ }^{s}\right)+\omega_{2} \alpha_{2}{ }^{s} \log \left(\alpha_{2}{ }^{s}\right)-\left(\omega_{1} \alpha_{1}{ }^{s}+\omega_{2} \alpha_{2}^{s}\right) \log \left(\omega_{1} \alpha_{1}{ }^{s}+\omega_{2} \alpha_{2}^{s}\right)}{\omega_{1} \alpha_{1}{ }^{s}+\omega_{2} \alpha_{2}{ }^{s}}
$$

and

$$
Z=\frac{\omega_{1} \beta_{1}{ }^{s} \log \left(\beta_{1}{ }^{s}\right)+\omega_{2} \beta_{2}{ }^{s} \log \left(\beta_{2}{ }^{s}\right)-\left(\omega_{1} \beta_{1}{ }^{s}+\omega_{2} \beta_{2}{ }^{s}\right) \log \left(\omega_{1} \beta_{1}{ }^{s}+\omega_{2} \beta_{2}{ }^{s}\right)}{\omega_{1} \beta_{1}{ }^{s}+\omega_{2} \beta_{2}{ }^{s}}
$$

Since the first factor in Eq. (37) is positive, the derivative is nonnegative whenever $Y \geqslant Z$. Setting $x_{i}=\alpha_{i}^{s}, y_{i}=\beta_{i}^{s}$, and $\omega_{1}=v$, $\omega_{2}=w$, and applying Lemma 10, this is always the case.

The proof of Proposition 4 also requires a lemma.

Lemma 11. If $M$ is an undominated finite set of preference pairs and $\left(\alpha_{1}, \alpha_{2}\right) \in M$, then there exists $\epsilon>0$ such that every other element of $M$ is dominated by either $\left(\alpha_{1}-\epsilon, 1\right)$ or by $\left(1, \alpha_{2}-\epsilon\right)$.

Proof of Lemma 11. Consider $\left(\beta_{1}, \beta_{2}\right) \in M$. Since $\left(\beta_{1}, \beta_{2}\right)$ does not dominate $\left(\alpha_{1}, \alpha_{2}\right)$, either $\alpha_{1}>\beta_{1}$, or $\alpha_{2}>\beta_{2}$. If $\alpha_{1}>\beta_{1}$, let $\delta=\left(\alpha_{1}-\beta_{1}\right) / 2$. Then $\left(\alpha_{1}-\delta, 1\right)$ dominates $\left(\beta_{1}, \beta_{2}\right)$. If $\alpha_{2}>\beta_{2}$, let $\delta=\left(\alpha_{2}-\beta_{2}\right) / 2$. Then $\left(1, \alpha_{2}-\delta\right)$ dominates $\left(\beta_{1}, \beta_{2}\right)$. Since $M$ is finite, this can be repeated for all remaining elements of $M$; let $\epsilon$ be the smallest such $\delta$, and every element of $M$ except $\left(\alpha_{1}, \alpha_{2}\right)$ is dominated either by $\left(\alpha_{1}-\epsilon, 1\right)$, or by $\left(1, \alpha_{2}-\epsilon\right)$.

Proof of Proposition 4. By Lemma 11, it suffices to show that for any $\epsilon$, the following pair of equations can be solved for $s$ and $\omega:$

$$
\begin{gathered}
\mathcal{P}_{s}\left(\alpha_{1}, \alpha_{2} ; 1, \omega\right)=\mathcal{P}_{s}\left(1, \alpha_{2}-\epsilon ; 1, \omega\right) \\
\mathcal{P}_{s}\left(\alpha_{1}, \alpha_{2} ; 1, \omega\right)=\mathcal{P}_{s}\left(\alpha_{1}-\epsilon, \alpha_{2} ; 1, \omega\right)
\end{gathered}
$$

If $s=0$ is a solution, then the proposition is proved. If $s \neq 0$, then the following equations must be solved: 


$$
\begin{gathered}
\alpha_{1}{ }^{s}+\omega \alpha_{2}{ }^{s}=\left(\alpha_{1}-\epsilon\right)^{s}+\omega \\
\alpha_{1}{ }^{s}+\omega \alpha_{2}{ }^{s}=1+\omega\left(\alpha_{2}-\epsilon\right)^{s}
\end{gathered}
$$

Solving (43) for $\omega$ yields

$$
\omega=\frac{1-\alpha_{1}^{s}}{\alpha_{2}{ }^{s}-\left(\alpha_{2}-\epsilon\right)^{s}}
$$

and plugging this into (42), and rearranging terms as below, gives the following that must be solved for $s$ :

$$
\left(1-\alpha_{1}^{s}\right)\left(1-\alpha_{2}^{s}\right)-\left[\alpha_{1}^{s}-\left(\alpha_{1}-\epsilon\right)^{s}\right]\left[\alpha_{2}^{s}-\left(\alpha_{2}-\epsilon\right)^{s}\right]=0
$$

$$
1-\alpha_{1}{ }^{s}-\alpha_{2}{ }^{s}-\left(\alpha_{1}-\epsilon\right)^{s}\left(\alpha_{2}-\epsilon\right)^{s}+\alpha_{1}{ }^{s}\left(\alpha_{2}-\epsilon\right)^{s}+\alpha_{2}{ }^{s}\left(\alpha_{1}-\epsilon\right)^{s}=0
$$

which can be rewritten as

$$
\begin{gathered}
1-e^{s \log \alpha_{1}}-e^{s \log \alpha_{2}}-e^{s \log \left(\left(\alpha_{1}-\epsilon\right)\left(\alpha_{2}-\epsilon\right)\right)}+e^{s \log \left(\alpha_{1}\left(\alpha_{2}-\epsilon\right)\right)} \\
+e^{s \log \left(\alpha_{2}\left(\alpha_{1}-\epsilon\right)\right)}=0
\end{gathered}
$$

Now, the first derivative of (47) with respect to $s$, evaluated at $s=0$, is 0 ; the second derivative is positive at $s=0$. It thus suffices to show that the left-hand side of (47) is negative for some $s<0$. Since $\alpha_{1}, \alpha_{1}-\epsilon \in(0,1)$ and $\epsilon>0$, we can set $A_{1}=\log \alpha_{1}$. Since $\log \left(\alpha_{1}-\epsilon\right)<\log \alpha_{1}<0$, there is some $\delta_{1}>0$ such that $A_{1}-\delta_{1}$ $=\log \left(\alpha_{1}-\epsilon\right)$. Similarly, we can define $A_{2}, \delta_{2}$ with $A_{2}=\log \alpha_{2}$ and $A_{2}-\delta_{2}=\log \left(\alpha_{2}-\epsilon\right)$.

Then consider the limit

$$
\lim _{s \rightarrow \infty} 1-e^{s A_{1}}-e^{s A_{2}}-e^{s\left(A_{1}-\delta_{1}+A_{2}-\delta_{2}\right)}+e^{s\left(A_{1}+A_{2}-\delta_{2}\right)}+e^{s\left(A_{2}+A_{1}-\delta_{1}\right)}
$$

which is the same as

$$
\lim _{t \rightarrow \infty} 1-e^{t\left|A_{1}\right|}-e^{t\left|A_{2}\right|}-e^{t\left(\left|A_{1}\right|+\left|A_{2}\right|+\delta_{1}+\delta_{2}\right)}+e^{t\left(\left|A_{1}\right|+\left|A_{2}\right|+\delta_{2}\right)}+e^{t\left(\left|A_{1}\right|+\left|A_{2}\right|+\delta_{1}\right)}
$$

Since this expression is dominated as $t \rightarrow \infty$ by the term $e^{t\left(\left|A_{1}\right|+\left|A_{2}\right|+\delta_{1}+\delta_{2}\right)}$, it must take on negative values for some $t>0$ (i.e., for some $s<0$ ). Therefore, the set of Eqs. (40) has a solution.

Proof of Proposition 5. Equality of overall preference is established by finding $s$ and $\omega$ such that

$$
\left(\frac{\alpha_{1}{ }^{s}+\omega \alpha_{2}{ }^{s}}{1+\omega}\right)^{1 / s}=\left(\frac{\beta_{1}{ }^{s}+\omega \beta_{2}{ }^{s}}{1+\omega}\right)^{1 / s}
$$

Although this is not defined at $s=0$, it is continuous through that point, and it suffices to consider

$$
\alpha_{1}^{s}+\omega \alpha_{2}^{s}=\beta_{1}{ }^{s}+\omega \beta_{2}^{s}
$$

Solving for $\omega$ gives

$$
\omega=\frac{\alpha_{1}^{s}-\beta_{1}^{s}}{\beta_{2}^{s}-\alpha_{2}^{s}}
$$

For $s \neq 0,(52)$ has a solution. This solution is positive since $\alpha_{1}>\beta_{1}$ and $\beta_{2}>\alpha_{2}$. For $s=0$, the solution, also positive, is

$$
\omega=\frac{\log \alpha_{1}-\log \beta_{1}}{\log \beta_{2}-\log \alpha_{2}}
$$

which can be found by taking the limit as $s \rightarrow 0$, or by solving $\alpha_{1} \alpha_{2}^{\omega}=\beta_{1} \beta_{2}^{\omega}$ directly.

Proof of Proposition 6. If $\epsilon<\alpha_{1}, \alpha_{2}$, then $\left(\alpha_{1}, \alpha_{2}\right),\left(1, \alpha_{2}-\epsilon\right)$, and $\left(\alpha_{1}-\epsilon, 1\right)$ are mutually undominated. Consider the case of a fixed $s<0$. By Proposition 5, there exist $\omega^{\prime}$ and $\omega^{\prime \prime}$ such that

$$
\mathcal{P}_{s}\left[\left(\alpha_{1}, \alpha_{2}\right) ; 1, \omega^{\prime}\right]=\mathcal{P}_{s}\left[\left(1, \alpha_{2}-\epsilon\right) ; 1, \omega^{\prime}\right]
$$

$$
\mathcal{P}_{s}\left[\left(\alpha_{1}, \alpha_{2}\right) ; 1, \omega^{\prime \prime}\right]=\mathcal{P}_{s}\left[\left(\alpha_{1}-\epsilon, 1\right) ; 1, \omega^{\prime \prime}\right]
$$

which can be solved for $\omega^{\prime}$ and $\omega^{\prime \prime}$ as follows:

$$
\begin{aligned}
& \omega^{\prime}=\frac{\alpha_{1}^{s}-\left(\alpha_{1}-\epsilon\right)^{s}}{1-\alpha_{2}^{s}} \\
& \omega^{\prime \prime}=\frac{1-\alpha_{1}^{s}}{\alpha_{2}^{s}-\left(\alpha_{2}-\epsilon\right)^{s}}
\end{aligned}
$$

We show first that there is an $\epsilon>0$ such that $\omega^{\prime}=\omega^{\prime \prime}$. To do this, we need to find $\epsilon$ such that

$$
\left[\alpha_{1}^{s}-\left(\alpha_{1}-\epsilon\right)^{s}\right]\left[\alpha_{2}^{s}-\left(\alpha_{2}-\epsilon\right)^{s}\right]=\left(1-\alpha_{1}^{s}\right)\left(1-\alpha_{2}^{s}\right)
$$

Note that the right-hand side of (58) is positive. As $\epsilon \rightarrow 0+$, the left-hand side of (58) goes to zero. As $\epsilon \rightarrow \min \left(\alpha_{1}, \alpha_{2}\right)$, the lefthand side of (58) tends to $+\infty$. Thus there is an $\epsilon$ $\in\left[0, \min \left(\alpha_{1}, \alpha_{2}\right)\right]$ for which $\omega^{\prime}=\omega^{\prime \prime}$. Let

$$
\begin{aligned}
& \left(\beta_{1}, \beta_{2}\right)=\left(1, \alpha_{2}-\frac{\epsilon}{2}\right) \\
& \left(\gamma_{1}, \gamma_{2}\right)=\left(\alpha_{1}-\frac{\epsilon}{2}, 1\right)
\end{aligned}
$$

Then

$$
\begin{array}{ll}
\mathcal{P}_{s}\left(\beta_{1}, \beta_{2} ; 1, \omega\right)>\mathcal{P}_{s}\left(\alpha_{1}, \alpha_{2} ; 1, \omega\right) & \forall \omega \leqslant \omega^{\prime} \\
\mathcal{P}_{s}\left(\gamma_{1}, \gamma_{2} ; 1, \omega\right)>\mathcal{P}_{s}\left(\alpha_{1}, \alpha_{2} ; 1, \omega\right) & \forall \omega \geqslant \omega^{\prime \prime}
\end{array}
$$

Since $\omega^{\prime}=\omega^{\prime \prime}$, the proposition is proved for the case where $s<0$.

By Proposition 3, increasing the value of $s$ will favor $\left(\beta_{1}, \beta_{2}\right)$ over $\left(\alpha_{1}, \alpha_{2}\right)$, and will also favor $\left(\gamma_{1}, \gamma_{2}\right)$ over $\left(\alpha_{1}, \alpha_{2}\right)$. This proves the proposition for all $s>-\infty$.

Note that if $s=-\infty$, as long as $\alpha_{1} \neq \alpha_{2}$, the undominated point

$$
\left(\min \left(\alpha_{1}, \alpha_{2}\right)+\frac{\left|\alpha_{1}-\alpha_{2}\right|}{2}, \min \left(\alpha_{1}, \alpha_{2}\right)+\frac{\left|\alpha_{1}-\alpha_{2}\right|}{2}\right)
$$

is always preferred over $\left(\alpha_{1}, \alpha_{2}\right)$.

Proof of Proposition 7. As in the proof of Proposition 5 it suffices to consider

$$
\alpha_{1}^{s}+\omega \alpha_{2}^{s}=\beta_{1}{ }^{s}+\omega \beta_{2}^{s}
$$

1. If $\alpha_{1}>\beta_{1}, \beta_{2}>\alpha_{2}$, then consider the following two limits:

$$
\begin{aligned}
& \lim _{s \rightarrow-\infty} \frac{\alpha_{1}{ }^{s}-\beta_{1}{ }^{s}}{\beta_{2}{ }^{s}-\alpha_{2}{ }^{s}}=0 \\
& \lim _{s \rightarrow \infty} \frac{\alpha_{1}{ }^{s}-\beta_{1}{ }^{s}}{{\beta_{2}}^{s}-\alpha_{2}{ }^{s}}=\infty
\end{aligned}
$$

Thus the range of the right-hand side of (52) is all positive $\omega$, and for every $\omega$ there is a degree of compensation $s$.

2. If $\alpha_{1}>\beta_{2}>\alpha_{2}>\beta_{1}$, then

$$
\mathcal{P}_{s}\left(\alpha_{1}, \alpha_{2} ; 1,1\right)>\mathcal{P}_{s}\left(\beta_{1}, \beta_{2} ; 1,1\right)
$$

Furthermore, for any $\omega \in(0,1)$,

$$
\begin{aligned}
\mathcal{P}_{s}\left(\alpha_{1}, \alpha_{2} ; 1, \omega\right) & >\mathcal{P}_{s}\left(\alpha_{1}, \alpha_{2} ; 1,1\right)>\mathcal{P}_{s}\left(\beta_{1}, \beta_{2} ; 1,1\right) \\
& >\mathcal{P}_{s}\left(\beta_{1}, \beta_{2} ; 1, \omega\right)
\end{aligned}
$$

Thus it is impossible to find a degree of compensation $s$ to achieve equality for all possible weights $\omega$.

Furthermore,

$$
\lim _{s \rightarrow-\infty} \frac{\alpha_{1}{ }^{s}-\beta_{1}{ }^{s}}{{\beta_{2}}^{s}-\alpha_{2}{ }^{s}}=\infty
$$




$$
\lim _{s \rightarrow \infty} \frac{\alpha_{1}{ }^{s}-\beta_{1}{ }^{s}}{{\beta_{2}}^{s}-\alpha_{2}{ }^{s}}=\infty
$$

and there is a minimal $\omega$ which is reached when

$$
\frac{d}{d s} \frac{\alpha_{1}^{s}-\beta_{1}^{s}}{\beta_{2}^{s}-\alpha_{2}^{s}}=0
$$

is equal to 0 , i.e., when

$$
\frac{\left(\beta_{2}{ }^{s}-\alpha_{2}{ }^{s}\right)\left(\alpha_{1}{ }^{s} \log \alpha_{1}-\beta_{1}{ }^{s} \log \beta_{1}\right)-\left(\alpha_{1}{ }^{s}-\beta_{1}{ }^{s}\right)\left(\beta_{2}{ }^{s} \log \beta_{2}-\alpha_{2}{ }^{s} \log \alpha_{2}\right)}{\left(\beta_{2}{ }^{s}-\alpha_{2}{ }^{s}\right)^{2}}=0
$$

\section{References}

[1] Hauser, J. and Clausing, D., 1988, “The House of Quality," Harvard Bus. Rev., 66(3), pp. 63-73.

[2] Saaty, T. L., 1980, The Analytic Hierarchy Process: Planning, Priority Setting, Resource Allocation. McGraw-Hill, New York.

[3] Koski, J. 1985, "Defectiveness of Weighting Method in Multicriterion Optimization of Structures," Commun. Appl. Numer. Methods, 1(6) pp. 333-337.

[4] Messac, A., 1996, "Physical Programming: Effective Optimization for Computational Design," AIAA J., 34(1), pp. 149-158.

[5] Athan, T. W., and Papalambros P. Y., 1996, "A note on weighted criteria methods for compromise solutions in multi-objective optimization," Eng. Optimiz., 27, pp. 155-176.

[6] Messac, A., Melachrinoudis, E., and Sukam, C. P., 2000, “Aggregate Objective Functions and Pareto Frontiers: Required Relationships and Practical Implications," Optim. Eng., 1(2), pp. 171-188.

[7] Messac, A., Sundararaj, G. J., Tappeta, R., and Renaud, J. E., 2000, Ability of Objective Functions to Generate Points on Non-Convex Pareto Frontiers," AIAA J., 38(6), pp. 1084-1091

[8] Messac, A., and Ismail-Yahaya, A., 2001, "Required Relationship Between Objective Function and Pareto Frontier Orders: Practical Implications," AIAA J., 39(11), pp. 2168-2174,

[9] Scott, M. J., 1999, Formalizing Negotiation in Engineering Design, Ph.D. thesis, California Institute of Technology, Pasadena, CA.

[10] Tribus, M., 1969, Rational Descriptions, Decisions, and Designs, Pergamon Press, New York.

[11] Otto, K. N., and Antonsson, E. K., 1991, "Trade-Off Strategies in Engineering Design,” Res. Eng. Des., 3(2), pp. 87-104,

[12] Scott, M. J., and Antonsson, E. K., 1998, "Aggregation Functions for Engineering Design Trade-offs," Fuzzy Sets Syst., 99(3), pp. 253-264,

[13] Wood, K. L., and Antonsson, E. K., 1989, "Computations With Imprecise Parameters in Engineering Design: Background and Theory," ASME J. Mech., Transm., Autom. Des., 111(4), pp. 616-625.

[14] Pareto, V., 1971, Manual of Political Economy, A. S. Schwier and A. N. Page, eds., Augustus M. Kelley, New York, (translated by Ann S. Schwier from the 1927 French Edition of Manuale di economia politica, 1906).

[15] Chen, W., Wiecek, M., and Zhang, J., 1999, "Quality Utility: A compromise Programming approach to robust design," J. Mech. Des., 121(2), pp. 179-187.

[16] Shan, S., and Wang, G. G., 2005, "An Efficient Pareto Set Identification Approach for Multi-Objective Optimization on Black-Box Functions, J. Mech. Des. 127(5), pp. 866-874.

[17] Hernandez, G., Simpson, T. W., Allen, J. K., Bascaran, E., Avila, L. F., and Salinas, F., 2001, "Robust Design of Families of Products with Production Modeling and Evaluation," J. Mech. Des., 123(2), pp. 183-190.

[18] Kim, H. M., Rideout, D. G., Papalambros, P. Y., and Stein, J. L., 2003, “Analytic Target Cascading in Automotive Vehicle Design,” J. Mech. Des., 125(3), pp. 481-489.

[19] Charles D. McAllister and Simpson, T. W., 2003, "Multidisciplinary Robust Design Optimization of an Internal Combustion Engine," J. Mech. Des., 125(1), pp. 124-130

[20] See, T.-K., Gurnani, A., and Lewis, K. 2004, "Multiattribute Decision Making
Using Hypothetical Equivalents and Inequivalents," ASME J. Mech. Des. 126(6), pp. 950-958

[21] Wassenaar, H. J., and Chen, W., 2003, "An Approach to Decision-Based Design With Discrete Choice Analysis for Demand Modeling," J. Mech. Des., 125(3), pp. 490-497.

[22] Wassenaar, H. J., Chen, W., Cheng, J., and Sudjianto, A., 2005, "Enhancing Discrete Choice Demand Modeling for Decision-Based Design," J. Mech. Des. (to appear).

[23] Wan, J., and Krishnamurty, S., 2001, "Learning-Based Preference Modeling in Engineering Design Decision-Making,” J. Mech. Des., 123(2), pp. 191-198.

[24] Tappeta, R. V., and Renaud, J. E., 2001, "Interactive Multiobjective Optimization Design Strategy for Decision Based Design,” J. Mech. Des., 123(2), pp. 205-215.

[25] Farhang-Mehr, A., and Azarm, S., 2003, “An Information-Theoretic Entropy metric for Assessing Multi-Objective Optimization Solution Set Quality. J. Mech. Des., 125(4), pp. 655-663.

[26] Maddulapalli, K., Azarm, S., and Boyars, A., 2005, "Interactive Product Design Selection With an Implicit Value Function," J. Mech. Des. (to appear).

[27] Olewnik, A., Brauen, T., Ferguson, S., and Lewis, K., 2004, "A Framework for Flexible Systems and its Implementation in Multiattribute Decision Making," J. Mech. Des., 126(3), pp. 412-419.

[28] Wood, W. H., and Agogino, A. M., 2005, "Decision-Based Conceptual Design: Modeling and Navigating Heterogeneous Design Spaces," ASME J. Mech. Des. 127(1), pp. 2-11.

[29] Franssen, Maarten P. M., Franssen, M. P. M., and Bucciarelli, L. L., 2004, "On Rationality in Engineering Design," ASME J. Mech. Des. 126(6), pp. 945 949.

[30] Law, W. S., and Antonsson, E. K., 1995, "Hierarchical Imprecise Design with Weights," Proc. of Fourth IEEE International Conference on Fuzzy Systems (FUZZ-IEEE/IFES'95) IEEE, New York, Vol. 1, pp. 383-388.

[31] Scott, M. J., and Antonsson, E. K., 1998, "Preliminary Vehicle Structure Design: An Industrial Application of Imprecision in Engineering Design," Proc. of 1998 ASME Design Engineering Technical Conferences, ASME, New York.

[32] Otto, K. N., 1992, "A Formal Representational Theory for Engineering Design," Ph.D. thesis, California Institute of Technology, Pasadena, CA.

[33] Keeney, R., and Raiffa, H., 1976, Decisions With Multiple Objectives: Preferences and Value Tradeoffs, Wiley, New York.

[34] Wood, K. L., Antonsson, E. K., and Beck, J. L., 1990, "Representing Imprecision in Engineering Design-Comparing Fuzzy and Probability Calculus," Res. Eng. Des., 1(3/4), pp. 187-203,

[35] Otto, K. N., and Antonsson, E. K., 1993, "The Method of Imprecision Compared to Utility Theory for Design Selection Problems," Design Theory and Methodology-DTM '93, ASME, New York, pp. 167-173.

[36] Dyckhoff, H. and Pedrycz, W., 1984, "Generalized means as model of compensative connectives," Fuzzy Sets Syst., 14, pp. 143-154.

[37] Scott, M. J., and Antonsson, E. K., 2000, "Using Indifference Points in Engineering Decisions," Proc. of 2000 ASME Design Engineering Technical Conferences and Computers and Information in Engineering Conference, ASME, New York, ASME Paper No. DETC2000/DTM-14559. 